\title{
Healthcare Utilization Among Patients Diagnosed with COVID-19 in a Large Integrated Health System
}

\author{
Brian Z. Huang, $P h D^{1,2}{ }^{(1)}$, Beth Creekmur, $M A^{7}$, Michael S. Yoo, MD³, \\ Benjamin Broder, MD, PhD ${ }^{4}$, Christopher Subject, $M D^{5}$, and Adam L. Sharp, MD
}

\begin{abstract}
'Department of Research and Evaluation, Kaiser Permanente Southern California, Pasadena, CA, USA; ${ }^{2}$ Department of Population and Public Health Sciences, Keck School of Medicine of USC, , CALos Angeles, USA; ${ }^{3}$ The Permanente Medical Group, Kaiser Permanente Santa Rosa Medical Center, Santa Rosa, CA, USA; 'S Southern California Permanente Medical Group, Baldwin Park Medical Center, Baldwin Park, CA, USA; ${ }^{5}$ Southern California Permanente Medical Group, Los Angeles Medical Center, Los Angeles, CA, USA; ${ }^{6}$ Departments of Clinical Science \& Health Systems Science, Kaiser Permanente Bernard J. Tyson School of Medicine, Pasadena, CA, USA.
\end{abstract}

BACKGROUND: The demands for healthcare resources following a COVID-19 diagnosis are substantial, but not currently quantified.

OBJECTIVE: To describe trends in healthcare utilization within 180 days for patients diagnosed with COVID-19 and identify patient factors associated with increased healthcare use.

DESIGN: Observational cohort study.

PATIENTS: A total of 64,011 patients with a testconfirmed COVID-19 diagnosis from March to September 2020 in a large integrated healthcare system in Southern California.

MAIN MEASURES: Overall healthcare utilization during the 180 days following COVID-19 diagnosis, as well as encounter types and reasons for visits during the first 30 days. Poisson regression was used to identify patient factors associated with higher utilization. Analyses were performed separately for patients who were and were not hospitalized for COVID-19.

KEY RESULTS: Healthcare utilization was about twice as high for hospitalized patients compared to nonhospitalized patients in all time periods. The average number of visits was highest in the first 30 days (hospitalized: 12.3 visits/30 person-days; non-hospitalized: 6.6) and gradually decreased over time. In the first 30 days, the majority of healthcare visits were telehealth encounters (hospitalized: 9.0 visits; non-hospitalized: 5.6 visits), and the most prevalent reasons for visits were COVIDrelated diagnoses, COVID-related symptoms, and respiratory-related conditions. For hospitalized patients, older age ( $\geq 65$ : RR $1.27,95 \%$ CI 1.15-1.41), female gender (RR 1.07, 95\% CI 1.05-1.09), and higher BMI ( $\geq 40$ : RR 1.07, 95\% CI 1.03-1.10) were associated with higher total utilization. For non-hospitalized patients, older age, female gender, higher BMI, non-white race/ethnicity, former smoking, and greater number of pre-existing comorbidities were all associated with increased utilization.

CONCLUSIONS: Patients with COVID-19 seek healthcare frequently within 30 days of diagnosis, placing high demands on health systems. Identifying ways to support patients diagnosed with COVID-19 while adequately

Received March 19, 2021

Accepted September 3, 2021

Published online January 6, 2022 providing the usual recommended care to our communities will be important as we recover from the pandemic.

KEY WORDS: COVID-19; SARS-CoV-2; utilization.

J Gen Intern Med 37(4):830-7

DOI: $10.1007 /$ s11606-021-07139-Z

(C) Society of General Internal Medicine 2021

\section{INTRODUCTION}

The global COVID-19 pandemic is straining our health systems and resources in unprecedented ways throughout the world. ${ }^{1,2}$ Most existing research focuses on the hospitalized COVID-19 population and the risks of critical illness or death, but there has been little reported about the outpatient and emergency department (ED) utilization patterns among those infected with COVID-19. To recognize the full extent of disease burden and prepare for ongoing needs of patients diagnosed with COVID-19, it is important to understand the utilization patterns among all COVID-19 patients, including those who are treated outside of the hospital. ${ }^{3}$

Not only is there little known about the overall burden of the COVID-19 pandemic on patients and health systems, but there is also a critical need to identify subgroups of patients who may require additional care following a COVID-19 diagnosis. ${ }^{4-6}$ Many risk factors, including age, sex, and obesity, ${ }^{7-10}$ have been identified as predictors of severe illness or death. Further assessment of the patients who require increased outpatient follow-up would be valuable in determining resource allocation, which is of particular importance during resourceconstrained periods of pandemic surge. In addition, understanding the types of care delivered and by which health professionals would inform future needs and efforts for improvement. This information may be particularly helpful in the subgroup of patients discharged after hospitalization, in whom recent studies have shown the prolonged impact of COVID-19 post-hospital discharge. ${ }^{11-13}$

Thus, the focus of this study was to describe the outpatient, $\mathrm{ED}$, and hospital utilization of healthcare following a confirmed COVID-19 diagnosis for a large, heterogeneous 
population of patients from an integrated health system. We characterized different patient groups and their utilization patterns to identify factors associated with the greatest healthcare needs following diagnosis. Additionally, we evaluated the types of visits, reasons for visits, and outcomes for these patients to help identify potential resource strain and understand the broader impact of this disease. As the COVID-19 pandemic continues to be a major health crisis worldwide with emerging variants and new surges, there is an urgent need to characterize the post-infection healthcare demands of COVID19 patients to better understand patient outcomes and properly allocate healthcare resources.

\section{METHODS}

\section{Study Design, Population, Settings}

This observational cohort study was conducted in the member population of Kaiser Permanente Southern California (KPSC), an integrated healthcare organization with more than 7500 physicians, 15 medical centers, and 231 medical offices. The KPSC system provides comprehensive healthcare to more than 4.6 million racially and socioeconomically diverse members residing within 7 counties of Southern California. KPSC coordinates care through regionwide electronic medical records (EMRs) that capture information on outpatient visits and inpatient stays received at KPSC-owned and KPSC-contracting facilities. The research database also includes administrative claims for members who receive out-of-network clinical care. All other patient data including demographics, lifestyle factors (e.g., smoking, body mass index $[\mathrm{BMI}]$ ), socioeconomic factors, and comorbidities are stored in the EMR. The study was approved by the KPSC Institutional Review Board.

\section{Inclusion and Exclusion Criteria}

This study included all KPSC members who received a SARS-CoV-2 positive nucleic acid amplification test between March 1 and August 31, $2020(N=67,354)$. Patients were excluded from the study if they were not KPSC members $(N=3338)$ or had an unknown gender $(N=5)$.

\section{Outcomes, Exposure, and Measures}

The primary outcome was overall healthcare utilization in the 180 days (in 30-day time intervals) following a test-confirmed COVID-19 diagnosis. All encounters occurring on the same day of the COVID-19 test were included. Patients were followed from the time of the COVID-19 test to 180 days after, death, or end of membership, whichever came first. To account for varying follow-up periods among patients, utilization was measured as the number of visits per 30 person-days.

Since most of the healthcare utilization occurred in the first 30 days following the COVID-19 diagnosis, we performed more detailed analyses on the visits during this first time window. Specifically, we evaluated the types of visits, such
Table 1 Patient Characteristics of Study Cohort of 64,011 Patients Diagnosed with COVID-19 from March 1 to August 31, 2020, Stratified by Hospitalization Status

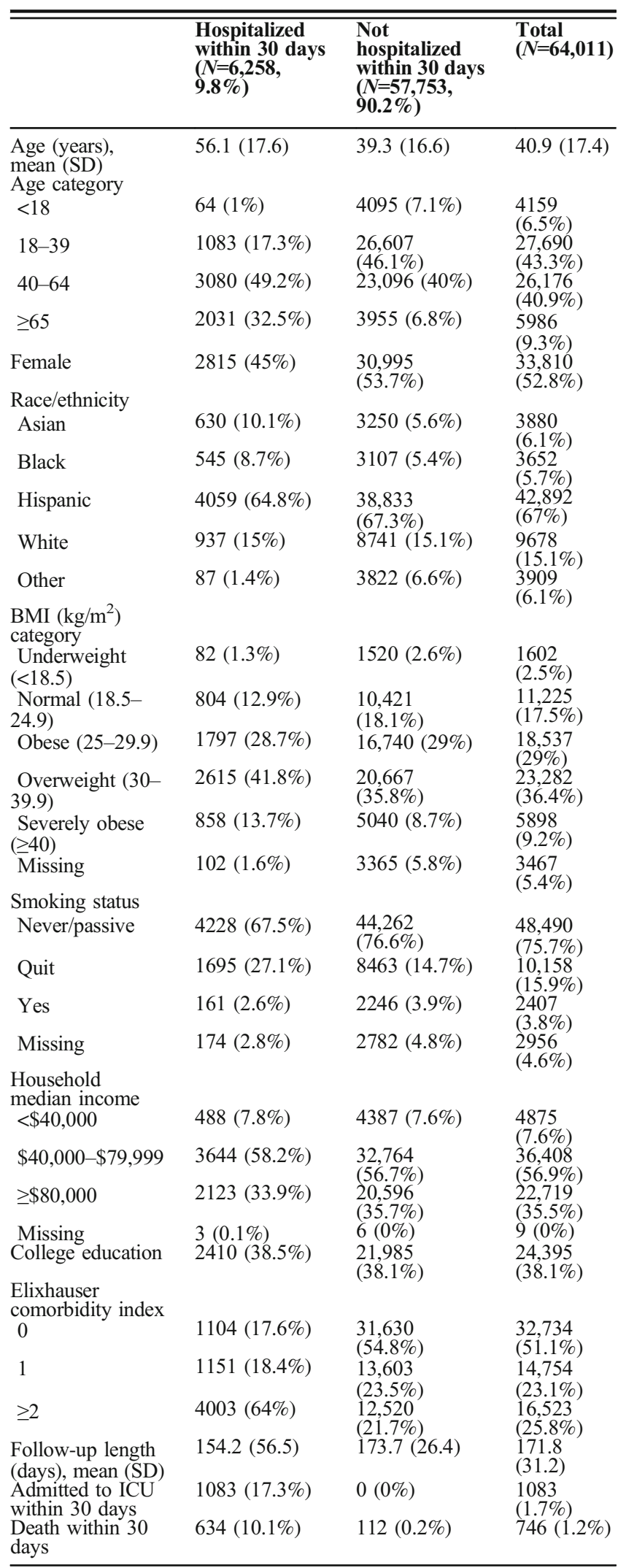

Abbreviations: SD, standard deviation; BMI, body mass index; ICU, intensive care unit 


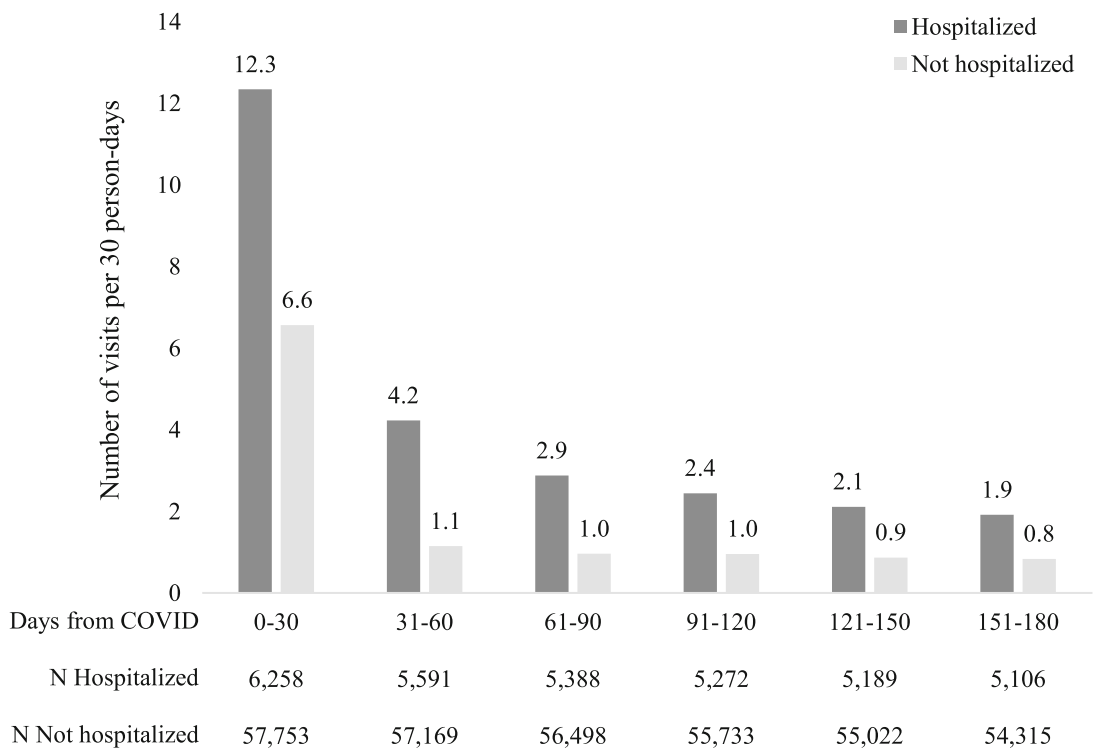

Figure 1 Average rates of overall utilization (number of total visits per 30 person-days) up to 180 days following COVID-19 diagnosis, by hospitalization status.

as telehealth (phone or videoconference appointments), inperson office visit, assisted care (e.g., hospice/skilled nursing facility), ED, urgent care, or hospitalization. For telehealth and in-person office visits, we further assessed the visit specialty (primary care or specialty care) and type of provider (physician, physician assistant/nurse practitioner, nurse, or mental health counselor).

In addition, we assessed the reasons for visits using the International Classification of Diseases, Tenth Revision, Clinical Modification (ICD-10-CM) codes associated with the visits in the first 30 days. ICD-10-CM codes were aggregated into clinically relevant body system categories (e.g., respiratory, circulatory, endocrine systems) using the Healthcare Cost and Utilization Project (HCUP) Clinical Classifications Software Refined (CCSR) for ICD-10-CM-coded diagnoses. ${ }^{14} \mathrm{COVID-related} \mathrm{di-}$ agnosis $\operatorname{codes}^{25}$ (Supplemental Table 1) were grouped into a separate category.

\section{Statistical Analysis}

We calculated the average rates (per 30 person-days) and $95 \%$ confidence intervals (CI) for the total number of visits in 30day intervals up to 180 days after COVID-19 diagnosis. For utilization in the first 30 days, we also calculated rates for the types of visits, visit specialty, type of provider, and reasons for visits. Rates for visit specialty and type of provider were only calculated for telehealth and in-person office visits.

For the total number and types of visits in the first 30 days, we calculated rates within subgroups defined by age category $(<18,18-39,40-64, \geq 65)$, gender, race/ethnicity (Asian, black, Hispanic, white, other), and BMI category (underweight $<18.5$, normal 18.5-24.9, overweight 25-29.9, obese 30-39.9, severely obese $\geq 40$ ).

Poisson regression was used to identify factors associated with higher utilization in the first 30 days. We estimated rate ratios (RR) and 95\% CIs for the association between overall utilization and age category, gender, race/ethnicity, BMI category, smoking status (current, former, never), income $(<\$ 40,000, \$ 40,000-79,999, \geq \$ 80,000)$, college education, and Elixhauser comorbidity index $(0,1, \geq 2)$. We included follow-up time as an offset in the model to account for varying observation times (due to censoring from death or end of membership during a given window).

Rate calculations and Poisson models were performed separately for patients who were and were not hospitalized within 30 days from the COVID-19 diagnosis. All analyses were conducted using SAS 9.4.

\section{RESULTS}

After exclusions, there were 64,011 patients who were diagnosed with COVID-19 from March 1 to August 31, 2020. The cohort had a mean age of 40.9 years and was comprised of $52.8 \%$ females and 67\% Hispanics (Table 1). Nearly 10\% ( $N=6258$ ) of the cohort was hospitalized within 30 days of COVID-19 diagnosis, of which $43.9 \%(N=2749)$ were admitted on the same day of the COVID-19 test and 17.3\% $(N=1083)$ were later admitted to the intensive care unit (ICU). Among hospitalized patients, the median length of stay was 5 days (interquartile range 2-9 days). Patients who were hospitalized were more likely to be older, male, obese, and former smokers compared to those who were not hospitalized.

Over $90 \%(N=58,571)$ of patients were followed until the end of the 180-day observation period. In each time interval, less than $2 \%$ of patients were censored due to death or end of membership. In the first 30 days following the COVID-19 diagnosis, $1.2 \%(N=746)$ of patients died and $0.8 \%(N=509)$ were lost to follow-up due to end of membership. During the 

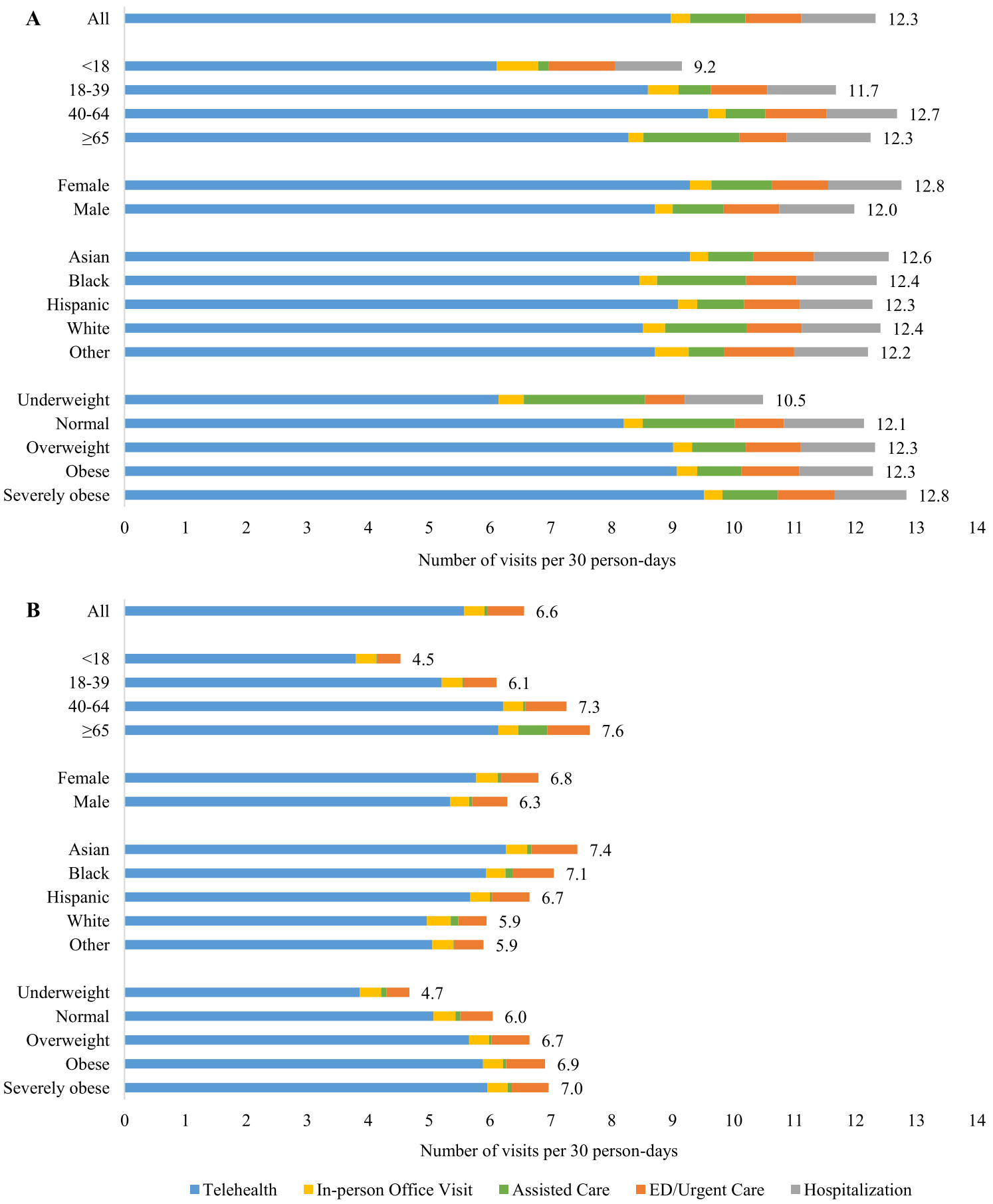

Figure 2 Average rates of overall utilization (number of total visits per 30 person-days) within 30 days after COVID-19 diagnosis among subgroups for A hospitalized and B non-hospitalized patients.

entire 180-day follow-up period, 1.5\% ( $N=969)$ of patients died and $7 \%(N=4471)$ became non-members.

For all patients, healthcare utilization was elevated in the first 30-day period after COVID-19 diagnosis and gradually decreased over time (Fig. 1). However, utilization rates were about twice as high for hospitalized patients compared to those for nonhospitalized patients in all time intervals. In the first 30 days following COVID-19 diagnosis, hospitalized patients had on average 12.3 total visits while non-hospitalized patients had on average 6.6 total visits. This decreased to 1.9 visits for hospitalized patients and 0.8 visits for non-hospitalized patients by the last 30-day time period (151-180 days after diagnosis).

When evaluating utilization in the first 30 days after COVID-19 diagnosis, telehealth encounters were the 


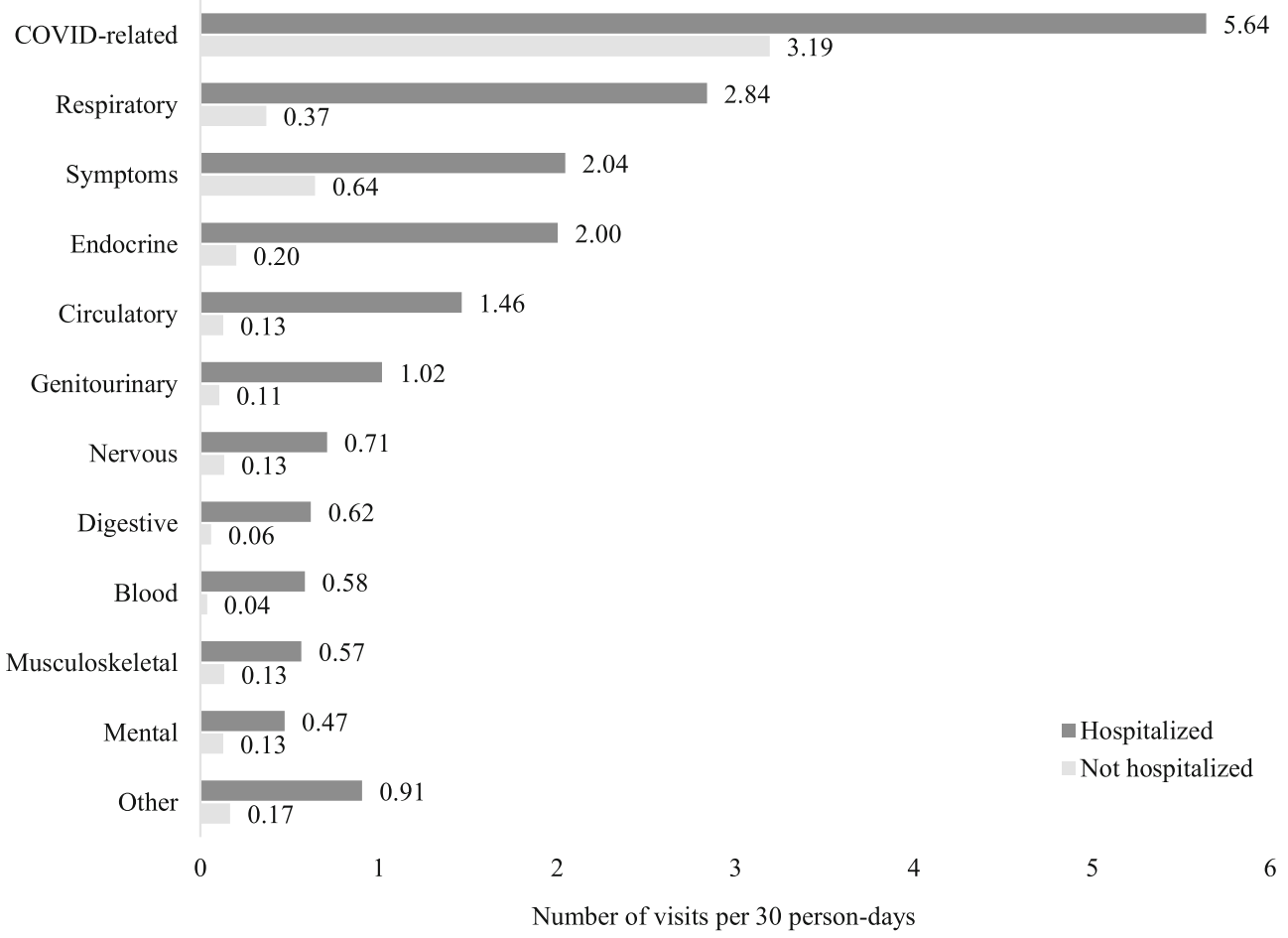

Figure 3 Average rates for the reasons for visit (number of visits per 30 person-days) within 30 days following COVID-19 diagnosis, by hospitalization status.

predominant type of visit for both hospitalized (9.0 visits/30 person-days) and non-hospitalized (5.6 visits/30 persondays) patients (Fig. 2/Supplemental Table 2). Furthermore, hospitalized patients had higher rates of assisted care $(0.9$ visits/30 person-days) and $\mathrm{ED} /$ urgent care ( 0.9 visits $/ 30$ person-days) visits compared to non-hospitalized patients $(0.1$ visits/30 person-days for assisted care, 0.6 visits $/ 30$ persondays for $\mathrm{ED} /$ urgent care).

Among the subgroups defined by age, sex, race/ethnicity, and BMI, overall utilization was highest among patients of older age and higher BMI and slightly higher for females compared to males for both hospitalized and non-hospitalized patients (Fig. 2/ Supplemental Table 2). Utilization rates did not appear to differ much across race/ethnicity for hospitalized patients (ranging from 12.2 to 12.6 visits/30 person-days), but were highest for Asians (7.4 visits/30 person-days) and lowest in whites (5.9 visits/30 person-days) for non-hospitalized patients. Utilization patterns for the type of visit were generally similar across subgroups, with telehealth encounters being the most prevalent (Fig. 2). Among hospitalized patients, rates of assisted care were higher for patients who were $\geq 65$, black, or white, or had underweight or normal BMI (Fig. 2).

For telehealth and in-person office visits, utilization patterns by visit specialty and provider type were similar for both hospitalized and non-hospitalized patients (Supplemental Table 3). For both groups, rates for primary care encounters were about twice as high as rates for specialty care encounters. In addition, patients were seen most frequently by physicians, followed by nurses, physician assistants/nurse practitioners, and mental health counselors (Supplemental Table 3).
In regard to reasons for visits, COVID-related diagnoses were the most common for both hospitalized (5.6 visits/30 person-days) and non-hospitalized (3.2 visits/30 person-days) patients (Fig. 3). Respiratory-related medical conditions, symptoms (e.g., cough, shortness of breath, fatigue), and endocrine-related medical conditions were the next most common diagnoses for both groups (Fig. 3).

Among hospitalized patients, older age (40-64: RR 1.31, 95\% CI 1.19-1.44; $\geq 65$ : RR 1.27, 95\% CI 1.15-1.41), female gender (RR 1.07, 95\% CI 1.05-1.09), and higher BMI ( $\geq 40$ : RR 1.07, 95\% CI 1.03-1.10) were associated with higher total utilization, while having a college education was associated with lower utilization (RR 0.986, 95\% CI 0.96-1.00) (Table 2). In contrast, among non-hospitalized patients, all of the patient characteristics were associated with either higher (older age, female, non-white race/ethnicity, higher BMI, former smoking, greater number of pre-existing comorbidities) or lower (higher household income, college education) total utilization (Table 2).

\section{DISCUSSION}

In this study, we examined healthcare utilization within 180 days following a COVID-19 diagnosis for a large and diverse population of patients from an integrated health system. We observed that hospitalized patients had twice the rates of utilization compared to non-hospitalized patients in all time intervals. Utilization rates were much higher in the first 30 days after COVID-19 diagnosis and steadily decreased over time. The highest rates of utilization were seen among older 
Table 2 Full Poisson Regression for the Associations Between Patient Characteristics and Total Number of Visits Within 30 Days Following COVID-19 Diagnosis, Stratified by Hospitalization Status

\begin{tabular}{|c|c|c|}
\hline & $\begin{array}{l}\text { Hospitalized } \\
\text { within } 30 \\
\text { days } \\
(N=6,258, \\
9.8 \%)\end{array}$ & $\begin{array}{l}\text { Not hospitalized within } 30 \\
\text { days }(N=57,753,90.2 \%)\end{array}$ \\
\hline & $\underset{C I}{\operatorname{RR}_{1}}(\mathbf{9 5 \%}$ & RR $(95 \% \text { CI })^{1}$ \\
\hline \multicolumn{3}{|l|}{ Age category } \\
\hline $\begin{array}{l}<18 \\
18-39\end{array}$ & $\begin{array}{l}1 \text { (ref) } \\
1.19(1.08,\end{array}$ & $\begin{array}{l}1 \text { (ref) } \\
1.32(1.29,1.34)\end{array}$ \\
\hline $40-64$ & $\begin{array}{l}1.31) \\
1.31(1.19 \\
1.44)\end{array}$ & $1.47(1.44,1.50)$ \\
\hline$\geq 65$ & $\begin{array}{l}1.27(1.15 \\
1.41)\end{array}$ & $1.44(1.41,1.47)$ \\
\hline Female & $\begin{array}{l}1.07(1.05 \\
1.09)\end{array}$ & $1.06(1.05,1.06)$ \\
\hline \multicolumn{3}{|l|}{ Race/ethnicity } \\
\hline White & 1 (ref) & 1 (ref) \\
\hline Asian & $\begin{array}{l}1.00(0.97, \\
1.03)\end{array}$ & $1.24(1.22,1.26)$ \\
\hline Black & $\begin{array}{l}0.99(0.96 \\
1.03)\end{array}$ & $1.15(1.13,1.16)$ \\
\hline Hispanic & $\begin{array}{l}0.98(0.96 \\
1.01)\end{array}$ & $1.13(1.11,1.14)$ \\
\hline Other & $1.00(0.93$, & $1.08(1.06,1.10)$ \\
\hline \multicolumn{3}{|l|}{ BMI $\left(\mathrm{kg} / \mathrm{m}^{2}\right)$} \\
\hline Underweight $(<18.5)$ & $\begin{array}{l}0.91(0.85, \\
0.98)\end{array}$ & $0.97(0.95,1.00)$ \\
\hline Normal (18.5-24.9) & 1 (ref) & 1 (ref) \\
\hline Obese $(25-29.9)$ & $\begin{array}{l}1.02(0.99, \\
1.05)\end{array}$ & $1.03(1.02,1.04)$ \\
\hline $\begin{array}{l}\text { Overweight }(30- \\
39.9)\end{array}$ & $\begin{array}{l}1.02(0.99, \\
1.04)\end{array}$ & $1.02(1.01,1.03)$ \\
\hline Severely obese $(\geq 40)$ & $\begin{array}{l}1.07(1.03 \\
1.10)\end{array}$ & $1.01(0.99,1.02)$ \\
\hline \multicolumn{3}{|l|}{ Smoking status } \\
\hline Never/passive & 1 (ref) & $1(\mathrm{ref})$ \\
\hline Quit & $\begin{array}{l}1.00(0.99, \\
1.02)\end{array}$ & $1.04(1.03,1.05)$ \\
\hline Yes & $\begin{array}{l}1.00(0.96, \\
1.05)\end{array}$ & $0.99(0.98,1.01)$ \\
\hline \multicolumn{3}{|l|}{$\begin{array}{l}\text { Household median } \\
\text { income }\end{array}$} \\
\hline$<\$ 40,000$ & $\begin{array}{l}0.92(0.89 \\
0.95)\end{array}$ & $1.00(0.98,1.01)$ \\
\hline$\$ 40,000-\$ 79,999$ & 1 (ref) & 1 (ref) \\
\hline$\geq \$ 80,000$ & $\begin{array}{l}1.00(0.99 \\
1.02)\end{array}$ & $0.97(0.96,0.98)$ \\
\hline College education & $\begin{array}{l}0.98(0.96 \\
1.00)\end{array}$ & $0.98(0.97,0.99)$ \\
\hline \multicolumn{3}{|l|}{$\begin{array}{l}\text { Elixhauser } \\
\text { comorbidity index }\end{array}$} \\
\hline 0 & 1 (ref) & $1(\mathrm{ref})$ \\
\hline 1 & $\begin{array}{l}1.01(0.99, \\
1.04)\end{array}$ & $1.13(1.12,1.14)$ \\
\hline$\geq 2$ & $\begin{array}{l}0.99(0.97, \\
1.01)\end{array}$ & $1.28(1.26,1.29)$ \\
\hline
\end{tabular}

${ }^{I}$ Rate ratio and $95 \%$ confidence interval

(aged $65+$ ) and severely obese (BMI $\geq 40$ ) patients. COVIDrelated diagnoses, symptoms, and respiratory-related medical conditions were the most common reasons for healthcare visits.

The burden of COVID-19 on healthcare resources is greater than recent pandemics involving influenza (H1N1) and other coronaviruses (SARS and MERS). ${ }^{15}$ Our results demonstrate a much greater magnitude of outpatients needs, as $99.6 \%$ of our cohort required at least one visit during the study period. By contrast, in previous flu seasons, $67.9 \%$ of influenza-like illness patient episodes led to a healthcare visit. ${ }^{16}$ Moreover, a prior study from Spain reported that healthcare utilization during the H1N1 pandemic was similar to that of the seasonal flu. ${ }^{17}$ Our findings also illustrate similarities to influenza in regard to decreases in productivity. ${ }^{18}$ However, COVID-19 appears to have a more severe and sustained impact, as shown by the high utilization rates in the first 30 days.

With social distancing measures and the continuing surges of new cases, the COVID-19 pandemic has forced drastic changes to our usual modes of clinical care. In our health system, use of telehealth services was considerably high, with telehealth visits comprising over $80 \%$ of all post-diagnosis visits in the first 30 days after COVID-19 diagnosis. Although we did not have a standardized telehealth follow-up program for COVID-19 patients across our health system, some individual medical centers did perform home monitoring for their patients to report test results and/or provide posthospitalization follow-up care. However, this would not have fully explained the high overall rates of telehealth encounters, as the home monitoring program was implemented for a small minority of our sample and would have only added approximately 1-2 visits for these patients. The elevated rates in the first 30 days compared to the final time period (151-180 days) further suggest that the increased utilization in the early period is likely driven by COVID-19 illness severity.

The rapid rise of telehealth during the pandemic has also been observed in other settings. Telehealth visits from the four largest US telehealth providers increased by $154 \%$ in the last week of March 2020 compared to the same time period in $2019 .{ }^{19}$ In addition, rates of telehealth visits increased 22-fold for a national sample of 16.7 million Medicare Advantage subscribers from January to June $2020{ }^{20}$ Telehealth will undoubtedly continue to play an important role during the pandemic by allowing providers to deliver care safely and widely while minimizing potential transmission. Importantly, it will help mitigate the burden on health facilities by reducing the use of in-person resources and essential supplies such as personal protective equipment. ${ }^{21}$

While Hispanic/Latinx comprise $45 \%$ of the KPSC member population, ${ }^{22}$ they made up $67 \%$ of our COVID-19 cohort. Higher rates of transmission and test positivity for the Hispanic and Latinx population have been observed previously due to socioeconomic factors such as working frontline service jobs, unemployment, and household size. ${ }^{23}$ Hispanic and Latinx populations have also been found to have higher risk of poorer outcomes from COVID-19 compared to whites in a recent study from Northern California. ${ }^{24}$ Our study showed that Hispanics and other non-whites had higher utilization compared to whites among non-hospitalized patients, but there was no difference among hospitalized patients. This suggests that patients had equal access to care and that minority 
populations were more likely to seek healthcare during the pandemic. As most of these visits were telehealth encounters, our findings indicate that telemedicine may be a potential strategy to reduce racial/ethnic inequities by improving access to care for disadvantaged groups.

The major strengths of this study are the large and diverse patient cohort and the comprehensive assessment of healthcare utilization following COVID-19 diagnosis. In particular, our study is one of the first to evaluate utilization for COVID-19 patients who were treated outside of the hospital, a population which has not been well-studied previously in the literature. As nearly $90 \%$ of patients were followed for the entire 180-day follow-up period, we were also able to assess the impact of COVID-19 on long-term healthcare utilization and compare patterns in the early and later phases of the disease. Additionally, our heterogeneous patient population allowed us to delineate utilization patterns across various demographic subgroups such as age, gender, and race. Using positive nucleic acid amplification lab results to identify COVID-19 patients increased the specificity of our case definition. However, we may have missed some patients who were diagnosed in the earlier period of the pandemic when testing was not as widely available, as well as patients who received a positive test outside of the KPSC health system. We also could not assess clinical status at the time of the COVID-19 diagnosis, as $87 \%$ of individuals were tested in outpatient settings (outpatient clinic or COVID-19 test clinics/ drive-thrus) and $76-80 \%$ were missing lab or vital sign data at the time of the visit. Finally, we could not evaluate the impact of clinical management on utilization due to the high variability across individual providers and medical centers and the evolution of clinical recommendations for treating COVID-19 over the pandemic. Though COVID-19 has not afforded the time to establish a standardized treatment approach, we expect that an interprofessional team-based primary care approach will be successful in caring for COVID-19 patients.

Our study showed that rates of healthcare utilization following a COVID-19 diagnosis are elevated for patients treated in both outpatient and inpatient settings, particularly among older and obese individuals. The high utilization of telehealth that we observed in this period further illustrates the direct changes in healthcare practices and clinical care brought on by COVID-19. Understanding these trends will allow health systems and providers to better determine patient needs and allocate appropriate resources as we recover from this worldwide pandemic.

Corresponding Author: Adam L. Sharp, MD; Department of Research and Evaluation, Kaiser Permanente Southern California, Pasadena, CA, USA (e-mail: Adam.L.Sharp@kp.org).

Supplementary Information The online version contains supplementary material available at https://doi.org/10.1007/s11606-02107139-z.
Author Contribution BZH-conception of study, analysis and interpretation of data, drafting and critical review of manuscript $B C$-interpretation of data, drafting and critical review of manuscript MSY - interpretation of data, critical review of manuscript $B B$-interpretation of data, critical review of manuscript CS-interpretation of data, critical review of manuscript ALS - conception of study, interpretation of data, drafting and critical review of manuscript

Funding This research was supported by internal funds provided by the Southern California Permanente Medical Group to the Care Improvement Research Team (CIRT). Brian Z. Huang is supported by grants from the National Cancer Institute at the National Institutes of Health (T32CA229110, K99CA256525).

\section{Declarations:}

Conflict of Interest: The authors declare that they do not have a conflict of interest.

\section{REFERENCES}

1. Annane D, Federici L, Chagnon J-L, et al. Intensive care units, the Achilles heel of France in the COVID-19 battle. The Lancet Regional Health - Europe. 2021:100046.

2. Hozhabri H, Piceci Sparascio F, Sohrabi H, et al. The Global Emergency of Novel Coronavirus (SARS-CoV-2): An Update of the Current Status and Forecasting. Int $J$ Environ Res Public Health. 2020;17(16):5648.

3. Donnelly JP, Wang XQ, Iwashyna TJ, Prescott HC. Readmission and Death After Initial Hospital Discharge Among Patients With COVID-19 in a Large Multihospital System. JAMA. 2021;325(3):304-306.

4. Ejaz H, Alsrhani A, Zafar A, et al.COVID-19 and comorbidities: Deleterious impact on infected patients. Journal of Infection and Public Health. 2020;13(12):1833-1839.

5. Ioannou GN, Locke E, Green P, et al. Risk Factors for Hospitalization, Mechanical Ventilation, or Death Among 10131 US Veterans With SARS-CoV-2 Infection. JAMA Network Open. 2020;3(9).

6. Zaki N, Alashwal H, Ibrahim S. Association of hypertension, diabetes, stroke, cancer, kidney disease, and high-cholesterol with COVID-19 disease severity and fatality: A systematic review. Diabetes \& Metabolic Syndrome. 2020;14(5):1133-1142.

7. Anderson MR, Geleris J, Anderson DR, et al. Body Mass Index and Risk for Intubation or Death in SARS-CoV-2 Infection: A Retrospective Cohort Study. Annals of Internal Medicine.

8. Bienvenu LA, Noonan J, Wang $\mathbf{X}$, Peter $\mathbf{K}$. Higher mortality of COVID19 in males: sex differences in immune response and cardiovascular comorbidities. Cardiovascular Research.

9. Dugail I, Amri EZ, Vitale N. High prevalence for obesity in severe COVID-19: Possible links and perspectives towards patient stratification. Biochimie. 2020;179:257-265.

10. Tartof SY, Gian L, Hong V, et al. Obesity and Mortality Among Patients Diagnosed With COVID-19: Results From an Integrated Health Care Organization. Annals of Internal Medicine. 2020;173(10):773-781.

11. Jiang DH, McCoy RG. Planning for the Post-COVID Syndrome: How Payers Can Mitigate Long-Term Complications of the Pandemic. $J$ Gen Intern Med. 2020;35(10):3036-3039.

12. Salehi S, Reddy S, Gholamrezanezhad A. Long-term Pulmonary Consequences of Coronavirus Disease 2019 (COVID-19): What We Know and What to Expect. $J$ Thorac Imaging. 2020;35(4):W87-W89.

13. Pero A, Ng S, Cai D. COVID-19: A Perspective from Clinical Neurology and Neuroscience. Neuroscientist. 2020;26(5-6):387-391.

14. Clinical Classifications Software Refined (CCSR). Healthcare Cost and Utilization Project (HCUP). Agency for Healthcare Research and Quality. 2020 (Rockville, MD): www.hcup-us.ahrq.gov/toolssoftware/ccsr/prccsr. jsp

15. Pitlik SD. COVID-19 Compared to Other Pandemic Diseases. Rambam Maimonides Med J. 2020;11(3):e0027.

16. Klepser DG, Corn CE, Schmidt M, Dering-Anderson AM, Klepser ME. Health Care Resource Utilization and Costs for Influenza-like Illness 
Among Midwestern Health Plan Members. Journal of managed care \& specialty pharmacy. 2015;21(7):568-573.

17. Galante M, Garin O, Sicuri E, et al. Health services utilization, work absenteeism and costs of pandemic influenza A (H1N1) 2009 in Spain: a multicenter-longitudinal study. PLoS One. 2012;7(2):e31696.

18. Keech M, Scott AJ, Ryan PJ. The impact of influenza and influenza-like illness on productivity and healthcare resource utilization in a working population. Occup Med (Lond). 1998;48(2):85-90.

19. Koonin LM, Hoots B, Tsang CA, et al. Trends in the Use of Telehealth During the Emergence of the COVID-19 Pandemic - United States, January-March 2020. MMWR Morb Mortal Wkly Rep. 2020;69(43):15951599.

20. Patel SY, Mehrotra A, Huskamp HA, Uscher-Pines L, Ganguli I, Barnett ML. Trends in Outpatient Care Delivery and Telemedicine During the COVID-19 Pandemic in the US. JAMA Intern Med. 2020.

21. Candel BGJ, Vaes SMM, van Bree EM, et al. Telemedicine in the emergency department to decrease personal protective equipment use: a before-and-after study. Emerg Med J. 2020.
22. Koebnick C, Langer-Gould AM, Gould MK, et al. Sociodemographic characteristics of members of a large, integrated health care system: comparison with US Census Bureau data. Perm J. 2012;16(3):37-41.

23. Chamie G, Marquez C, Crawford E, et al.SARS-CoV-2 Community Transmission disproportionately affects Latinx population during Shelterin-Place in San Francisco. Clin Infect Dis. 2020.

24. Jacobson M, Chang T, Shah M, Pramanik R, Shah SB. Racial and Ethnic Disparities in SARS-CoV-2 Testing and COVID-19 Outcomes in a Medicaid Managed Care Cohort. American Journal of Preventive Medicine. 2021.

25. Center for Disease Control and Prevention. ICD-10-CM Official Coding and Reporting Guidelines April 1, 2020 through September 30, 2020 , Available from: https://www.cdc.gov/nchs/data/icd/COVID-19-guidelines-final.pdf (Accessed 20 Sept 2020).

Publisher's Note: Springer Nature remains neutral with regard to jurisdictional claims in published maps and institutional affiliations. 